\title{
Analysis of a factory of the future using an integrated set of software for manufacturing systems modeling
}

\author{
Masami Shimizu \\ David Van Zoest \\ Manufacturing Systems Engineering \\ University of Wisconsin - Madison \\ 164 Mechanical Engineering Building \\ 1513 University Avenue \\ Madison, Wisconsin 53706
}

\section{ABSTRACT}

Design of a green-field factory, termed a 'factory of the future', proposed by an electrical equipment manufacturer was analyzed using an integrated set of software for manufacturing systems modeling.

The analysis involved the following four stages. First, a large data base containing part dimensions and processing conditions was analyzed using a spreadsheet software (Lotus 1-2-3) to generate a modeling data base. Second, a rough-cut analysis of each manufacturing line in the factory was performed utilizing queuing models (MANUPLAN In) to yield information regarding bottleneck stations, work-in-process levels, and part flow times. Third, detailed analysis for the entire factory with emphasis on the effects of job release dates and job scheduling rules was conducted by applying simulation models (SIMAN) which were rapidly created by a codegeneration software (SimStarter). Finally, graphical animation of the entire factory was created (CINEMA) providing the company's design project team with a visual aid in understanding the factory they were designing.

The analysis procedure, from data base manipulation to graphical animation, was integrated by software linkages. This integration enabled the analysis team to complete their work in a short time frame, and provided valuable feedback to the factory design team. Thus, utilizing this integrated approach is expected to be beneficial to similar manufacturing design and analysis projects.

\section{BACKGROUND}

A Wisconsin-based electrical equipment company currently manufactures their products in a plant that has undergone numerous additions required to accommodate growth in product demand. These additions of separate facilities for manufacturing processes have led to an inefficient manufacturing sequence. As a result, the company's current manufacturing facility has many inherent problems including: high work-in-process (WIP), long manufacturing lead time, and excessive material handling. In order to remain competitive in the market the company has decided to build a new facility, termed 'the factory of the future'. The new facility will incorporate a flow line concept coupled with increased automation and the Just-In-Time philosophy to resolve the problems mentioned above.

A team of faculty members and graduate students from the Manufacturing Systems Engineering Program at the University of Wisconsin - Madison (UW) was given an opportunity to participate in this factory of the future project. The company was in the developmental stage of the facility which created an excellent opportunity for investigating the feasibility of their plans and goals using manufacturing systems modeling and analysis. This paper, by two members of the team, presents the analysis methodology used by the team, results of the analysis, and the benefits and experience gained from using an integrated set of software for manufacturing systems modeling.

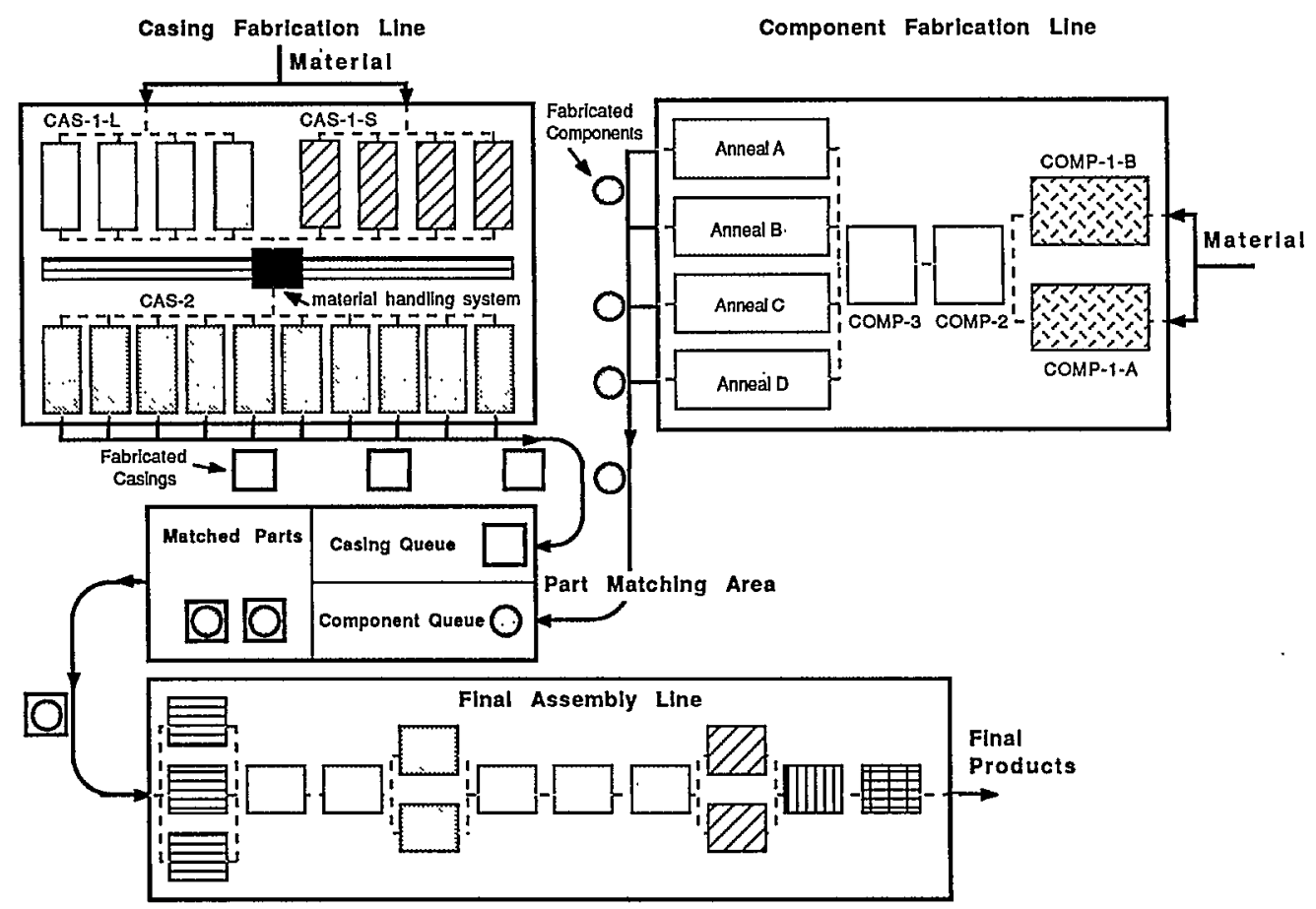

Figure 1 : Layout of the Proposed Facility 


\section{A FACTORY OF THE FUTURE}

A schematic diagram of the proposed new factory is presented in Figure 1. The factory consists of two fabrication lines for two major parts, 'casings' and 'components' (the actual names are withheld for confidentiality), and one final assembly line. The planned factory is characterized by the following features. First, parallel fabrication lines with different system configurations for the two main parts are connected to one final assembly line. Second, part matching of an identical order number is required for fabricated parts to begin the final assembly. Third, a capacity limitation exists in this part matching area. Finally, batch production is performed at the part fabrication lines whereas a unit-lot-size (lot size of one) production is performed at the final assembly line.

After discussing the above features and associated problems of the factory with the company's project members, the UW team set the following analysis objectives: 1) analyze the production capacity of each manufacturing line, 2) estimate the WIP in both the manufacturing lines and the part matching area, 3) evaluate the effects of scheduling rules for minimizing flow time and job lateness, and 4) analyze the effects of job release dates on WIP in the part matching area.

\section{METHOD FOR MODELING AND ANALYSIS: AN INTEGRATED APPROACH}

In order to meet the objectives, the team used an integrated set of software for manufacturing systems modeling. [Anderson et al. (1988), Shimizu (1988)] This analysis approach is based on linkages of five PC-based software packages, that is, Lotus 1-2-3 1 , MANUPLAN II, SimStarter ${ }^{2}$, SIMAN, and CINEMA ${ }^{3}$. Lotus 1-23 performs engineering calculation and data base manipulation, MANUPLAN II peiforms rough-cut dynamic analysis based on queuing theory, SimStarter converts MANUPLAN queuing models into SIMAN simulation models, SIMAN performs detailed simulation of the factory, CINEMA provides graphical animation of the factory. (Figure 2)

1. SPREADSHEET

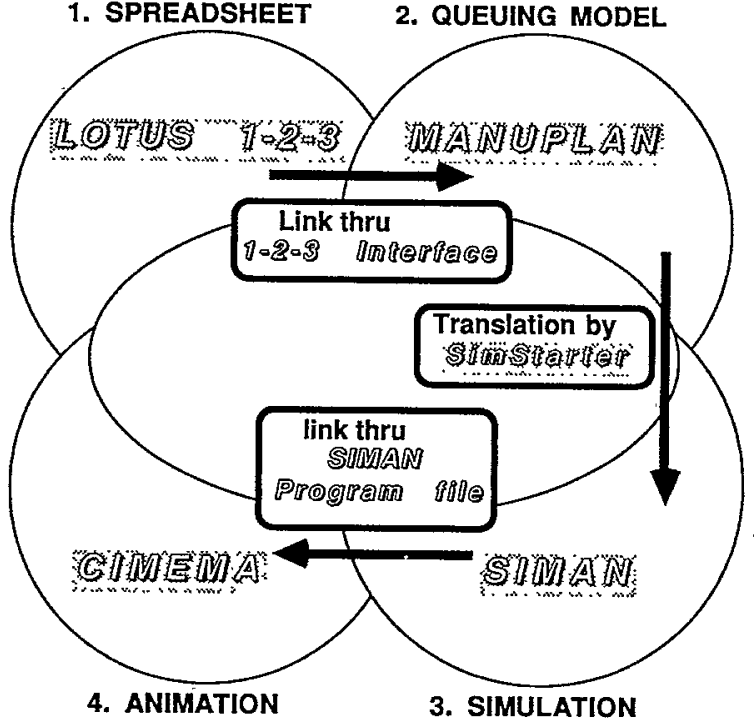

Figure 2: An Integrated Set of Software for Manufacturing Systems Modeling

\footnotetext{
1 Lotus 1-2-3 : A product of Lotus Development Corporation

2 MANUPLAN II and SimStarter : Products of Network Dynamics Inc.

3 SIMAN and CINEMA: Products of Systems Modeling Corporation
}

This integrated set of modeling tools met all of the requirements of the analysis team. First, the team needed to perform both rough-cut and detailed analysis to determine if the new factory would meet the company's expectations. The integrated modeling tool was suitable for this requirement because it covered the analysis ranging from rough-cut level to detailed level. Second, the team was given a very short time frame to complete the analysis, since the company had a tight implementation schedule. Rapid modeling via the software linkages helped in clearing this constraint by minimizing modeling time. [Anderson et al. (1988), Suri and Tomsicek (1988)] Third, some of the team members were not familiar with such modeling tools. Ease of use of the integrated software enabled these team members to use the tools with a very little training.

The following procedure outlines the team's specific approach to modeling and analyzing the new factory using the integrated modeling tools.

1) Modeling data preparation: Product types, production demands, part dimensions and part processing conditions were obtained from the company by interviewing the company's engineers. The team utilized this information to revise an existing data base. With this data, the team calculated the setup and processing times of manufacturing processes for each product type by using Lotus 1-2-3. This procedure yielded a basic data base for subsequent modeling.

2) Rough-cut analysis: The team utilized MANUPLAN to investigate the capacity, WIP, and flow time of the part fabrication lines and the final assembly line. [Suri et al. (1986), Suri (1988)] The MANUPLAN model was created by linking a MANUPLAN model input area with the previously developed Lotus 1-2-3 modeling data base via cell numbers of the Lotus 1-2-3 spreadsheet. (MANUPLAN II uses Lotus 1-2-3 as its user interface.)

3) Detailed analysis of the entire factory: The team examined the factory as a whole via simulation methods. Job release dates and scheduling rules were investigated with emphasis placed on their impact in achieving the company's goals, for example, minimizing WIP and manufacturing lead time. For this analysis, a SIMAN simulation model [Pegden (1987)] was created from the previous MANUPLAN model via the SimStarter translation software. [Suri and Tomsicek (1988)] The initially created simulation model was then modified to incorporate further design and factory operating details to study the above issues.

4) Graphical-animation: Graphical animation was employed to verify the analysis and to present the analysis results visually to company representatives. A CINEMA graphical animation was created by using the previous SIMAN simulation model linked to a separatelycreated CINEMA layout file. [Miles and Sandowski (1988)]

5) Recommendations: Finally the team summarized the results of the study by forwarding recommendations regarding implications of the company's current view of the factory.

\section{ROUGH-CUT ANALYSIS}

\subsection{MODELING DATA PREPARATION}

As described, the basic data for modeling was obtained from the company. There was, however, a major difficulty in utilizing this data. There were 80 types for the components and 120 types for the casings. Such diversity of part types created a wide variation in fabrication and assembly times. The large data base clearly increased the size and complexity of the model as well as the analysis and computation time. Therefore the team decided to aggregate the data base to make the modeling work manageable and to allow completion of the analysis within a limited time frame. With this scenario, the data base was consolidated into five types based upon final assembly characteristics, and setup and processing times could then be aggregated by calculating weighted average times based on the product demands. Such preparation of a modeling data base greatly simplified future modeling activities without compromising accuracy of the analysis, and proved invaluable in completing the project within the required time frame. 
Using the aggregated data base, MANUPLAN models were created to perform rough-cut analysis for the respective manufacturing lines. MANUPLAN yields steady state estimates of machine utilization, WIP, and part flow time based on dynamic analysis of the systems. The purpose of this stage of analysis is to determine if each manufacturing line has an adequate production capacity and meets its WIP and flow time objectives, before integrating the lines to model the overall factory . [Suri (1988)] We now describe this analysis and the insights gained for two part fabrication lines.

\subsection{THE COMPONENT FABRICATION LINE}

\subsubsection{DESCRIPTION OF FABRICATION PROCESS (Component line)}

Manufacture of the components consists of four major operations. As shown in Figure 1, the first and fourth operation have multiple resources whereas the remaining operations have only single resources. It should be noted here that part processing time for the first three operations are less than 10 minutes per part while the fourth operation (annealing) requires approximately one half day. The resulting flow time for the components is quite long compared with that of the casings.

Of key interest in this manufacturing line are the first and the fourth processing stations. Each of these processing stations is partially dedicated to a certain range of the product line due to its machine capacity for material sizes. This fact has an impact on the utilization levels of the machines. Dedications are as follows:

-- 1st processing station --

COMP-1-A: Largest 75\% of part types

COMP-1-B: Smallest $75 \%$ of part types

-- 4th processing station --

Anneal A: Parts with material width $W$

Anneal B: Parts with material width $X$

Anneal C: Parts with material width $Y$

Anneal D: Parts with material width Z

Modeling the above machine dedication was easily accomplished in MANUPLAN which has an input parameter to allocate percentages of an operation to different equipments.

MANUPLAN UTILIZATION REPORT

( COMPONENT FABRICATION LINE - INITIAL CONFIG. )

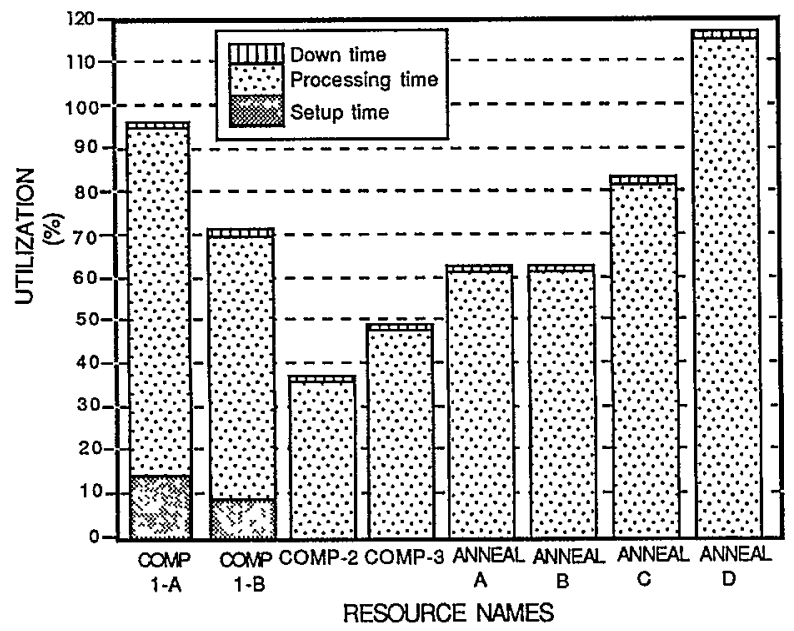

Figure 3: Utilization Summary Report for the Compontent Fabrication Line ( The Initial Configuration)

\subsubsection{ANALYSIS RESULTS FOR THE PROPOSED SYSTEM (Component line)}

Figure 3 shows a MANUPLAN equipment utilization summary for the system configuration under review at the time this study began. It shows that, under the initially proposed design configuration, the desired production could not be achieved. The bottleneck areas of production were COMP-1-A of the first processing station and Anneal D of the fourth processing station. The other machines were either at or significantly below the utilization limit.

\subsubsection{INSIGHTS AND RECOMMENDATIONS (Component line)}

Motivated by the capacity deficiency in the production line, the team pursued potential alternatives. Since this study was based on early estimates of manufacturing processes, the team investigated what changes in operation specifications would be required to make the desired production achievable. Resultant findings could provide a focus for process improvements over the next few months.

At the first and fourth stations, it was apparent that bottlenecks were being caused by load imbalance across the machines (see Figure 3) Using MANUPLAN's rapid 'what-if' capability (about a minute for each 'what-if'), the team found that the company's desired production levels could be achieved if utilization of the first and the fourth resources could be balanced by changing machine dedication conditions. This result is shown in Figure 4.

With respect to setup time, lot size and down time, as Figure 4 shows, the team found that: 1) setup time reduction had little impact on the overall capacity of the line, 2) lot size increases, accordingly, showed little benefit, and 3) since fairly good machine dependability was assumed, little room for improvement existed in this area.

The company's initial expectation prior to this analysis was that setup time reduction would prove an important factor in increasing the performance of their new factory. The company, however, had already reduced setup time considerably. Our analysis revealed that the need for balanced machine dedication was much more important than further setup time reduction and that achieving the desired production would only be feasible if all machines in the two bottleneck stations could be fully utilized.

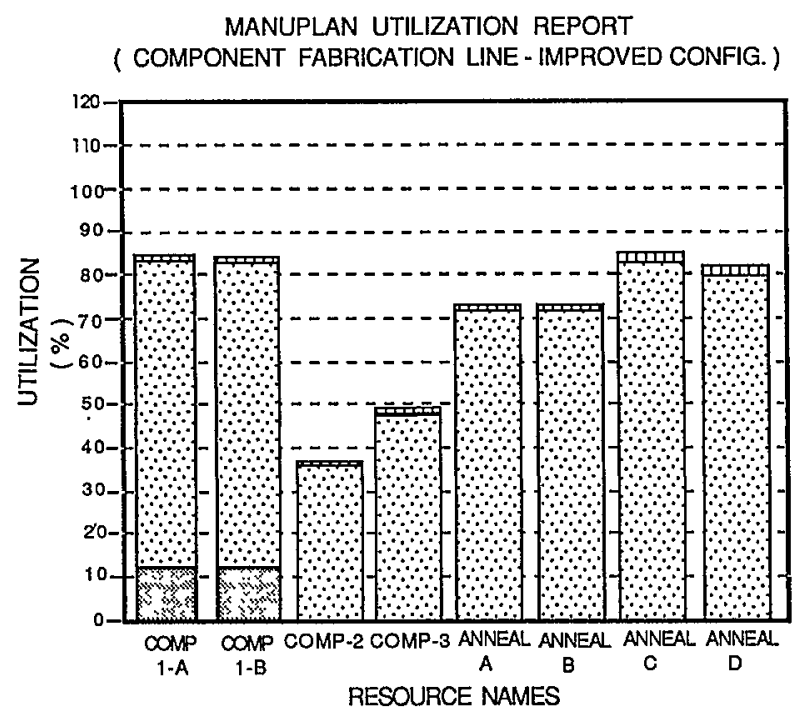

Figure 4: Utilization Summary Report for the Compontent Fabrication Line ( The Improved Configuration ) 


\subsubsection{THE CASING FABRICATION LINE}

\subsubsection{DESCRIPTION OF FABRICATION PROCESS (Casing line)}

Manufacturing of the casings consists of two primary operations. The first operation requires eight machines with four of them dedicated to large material sizes (CAS-1-L) and the other four dedicated to small material sizes (CAS-1-S). The second operation utilizes ten machines capable of processing all material sizes (CAS-2) and therefore undedicated. The required machine dedications were modeled similar to the component fabrication line.

\subsubsection{ANALYSIS RESULTS FOR THE PROPOSED SYSTEM (Casing line)}

Once again, our analysis showed that the desired production level could not be achieved by the initially proposed system configuration. Figure 5 illustrates the individual machine utilizations as calculated for the proposed system.

As shown in Figure 5, the first operation is the bottleneck for the proposed system. A significant load imbalance exists between the CAS-1-L and the CAS-1-S. Also, a large amount of down time exists in both CAS-1-L\&S and CAS-2.

\subsubsection{INSIGHTS AND RECOMMENDATIONS (Casing line)}

After sharing the results with the company's engineers, a number of changes were suggested as performance improvements within the casing fabrication line. The most feasible alternative, and therefore the basis for recommendation to the company was determined via quick 'what-if' analysis using MANUPLAN. The suggestions implemented were:

1) Implement an intensive preventative maintenance program to improve the reliability of machines with the aim of increasing mean time to failure by $130 \%$.
2) Substitute one CAS-1-L for one CAS-1-S to balance the machine loads

3) Aim to reduce processing times by ten percent across the board.

If these three recommendations are followed, then the company will reach the desired production capacity in the casing fabrication line. Figure 6 illustrates the resulting machine utilization for this case.

\section{DETAILED ANALYSIS USING SIMULATION}

For the next step in our analysis, detailed analysis of the entire factory was performed. Our motivation for this stage of analysis is as follows.

1) The company's intention for the new facility is that, in addition to the superior product and factory design, the entire factory must be operated by a 'just-in-time' type of approach through an efficient production control scheme.

2) Serious concerns exist about the WIP amount in the part matching area because of a buffer size limitation which must be strictly observed.

3) Although individual manufacturing lines have been analyzed extensively, the behavior of the factory as a whole has not yet been researched.

In order to clarify the above issues, a simulation model for the entire factory was developed. The aim of the simulation mode was, more specifically, to study the effects of two key factory operation factors: 1) job release dates for part fabrication, and 2) scheduling policy for job sequencing. Due to paper length limitations, we present only our study of job release dates, although the simulation modeling procedure is fully described for the both of the above issues.
MANUPLAN UTILIZATION REPORT (CASING FABRICATION LINE - INITIAL CONFIG.)

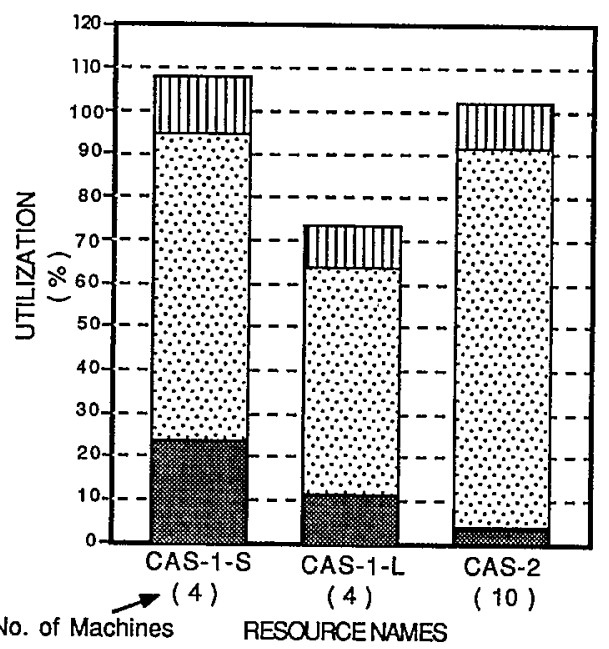

Figure 5: Utilization Summary Report for the Casing Fabrication Line ( The Initial Configuration )
MANUPLAN UTILIZATION REPORT (CASING FABRICATION LINE - IMPROVED CONFIG.)

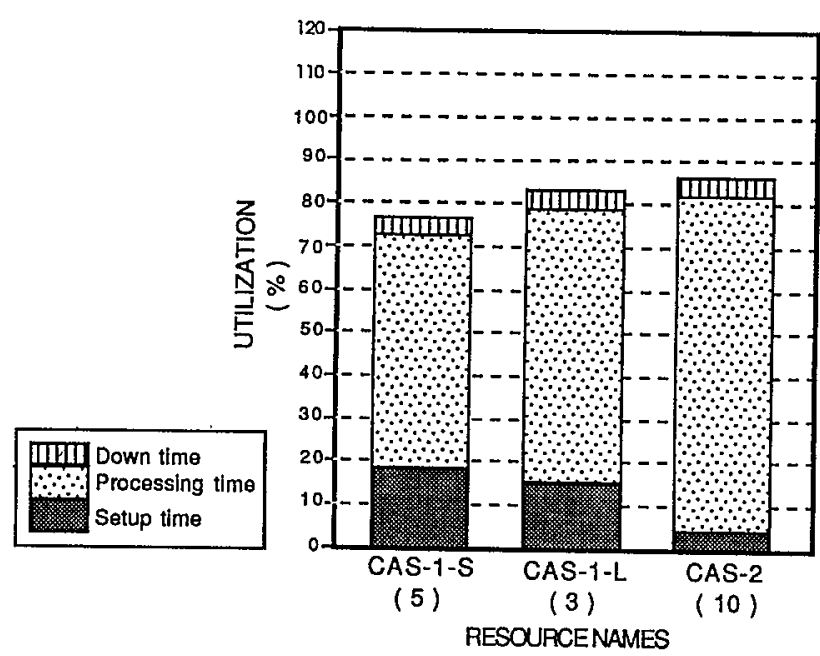

Figure 6: Utilization Summary Report for the Casing Fabrication Line ( The Improved Configuration ) 


\subsection{RAPID SIMULATION MODEL BUILDING OF THE ENTIRE FACTORY}

In order to perform a detailed analysis of the above issues, the simulation model needed to incorporate the following features:

1) Part grouping of fabricated parts to begin the final assembly (Connection of the part fabrication lines with the final assembly line)

2) Assignment of job order numbers to dispatching lots for part fabrication

3) Due date setting for the dispatching lots

4) Job release delay for the casing fabrication (Delay in starting the casing fabrication due to flow time differences between components and casings)

5) Matching of the fabricated parts with identical job order numbers at the part matching area.

6) Lot size change at the beginning of the final assembly line

7) Scheduling parameter calculation at each processing station

8) Job order sequencing at each processing station based on four scheduling rules, First-In-First-Out, Due-Date-First, Slack, and Critical Ratio

As described in a previous section, the SIMAN simulation language was chosen for the simulation study. Also, in keeping with our integrated software methodology, the SimStarter program was used to translate the already-developed MANUPLAN models into SIMAN models. Specifically, the following three steps were taken to build a simulation model with the above features.

1) First, the three MANUPLAN models of the individual lines (the casing, component, and final assembly lines) were combined into one MANUPLAN model file. Note that the three lines were not yet connected, but just resided as three separate lines in one MANUPLAN model.

2) Second, this MANUPLAN model was translated into SIMAN source code by SimStarter.

3) Finally, the SIMAN simulation model generated by SimStarter was modified to incorporate all the above-described features.

This procedure enabled a simulation model of the entire factory to be developed in only two days (see section 7), which is in sharp contrast to one or two months often expected for such a task.

\subsection{ANALYSIS OF EFFECTS OF JOB RELEASE DATES}

As Figure 7 shows, production of the electrical equipment is triggered by customer order entry, which in turn triggers a job release order to manufacture components, and after some delay (job release delay) a job release order to manufacture casings. The job release delay is necessary since the manufacturing time for the components is substantially longer than that of the casings (due to annealing time). After fabrication, both of the parts wait in a matching area until job number matching between two parts occurs. The matched parts are then grouped and fed into the final assembly, and are hopefully completed by the due date.

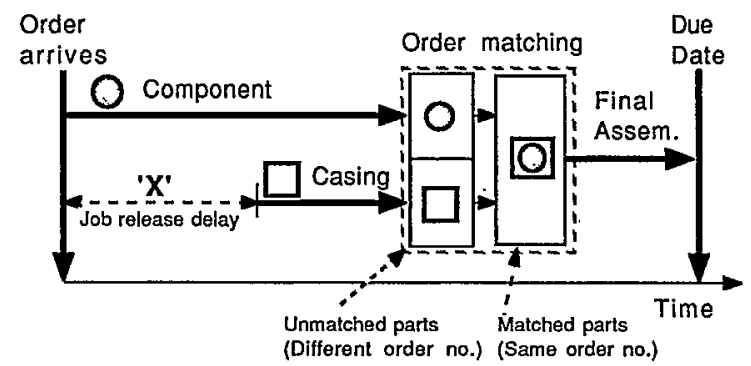

Figure 7: Job release delay and order matching
The amount of job release delay, indicated by ' $\mathrm{X}$ ' in Figure 7 , is an important parameter in the process flow. Appropriate selection of this parameter is critical in maintaining the optimal situation in the part matching area. Instead of studying the job release delay individually for each product type, it was decided to use a single parameter to identify the delay. In other words, a percentage of the difference in processing times for the two fabricated parts was used to represent the amount of delay in the analysis. The percentage is denoted by RDF (Release Delay Factor) in the equation below. With this single parameter, the job release delay time for each product (characterized by assembly types as explained before) is expressed by the following equation.

$$
\begin{aligned}
& \mathrm{X}=\mathrm{RDF} \times\left(\sum \mathrm{TCOMP}-\Sigma \mathrm{TCAS}\right) \\
& \text { where, } \\
& \mathrm{X}: \text { release delay time for part type } \mathrm{j} \text { (min.) } \\
& \mathrm{RDF}: \text { release delay factor }(\%) \\
& \Sigma T C O M P: \text { total processing time of component type } \mathrm{j} \text { (min.) } \\
& \sum \mathrm{TCAS}: \text { total processing time of casing type } \mathrm{j} \text { (min.) } \\
& \mathrm{j}: \text { part type } 1-5 \text { based on assembly characteristics }
\end{aligned}
$$

The RDF was varied from $0 \%$ to $150 \%$ for the analysis. In order to evaluate the effect of RDF, the WIP amount in the part matching area was chosen and measured in the SIMAN simulation program.

\subsection{RESULTS OF THE SIMULATION ANALYSIS}

Figure 8 shows the results of simulation analysis for different RDF values. The result shows that:

1) Releasing casings too early, i.e. RDF $\leq 25 \%$, will cause a large amount of casing WIP in the matching area

2) Releasing casings too late, i.e. $\mathrm{RDF} \geq 75 \%$, will cause a large amount of component WIP in the matching area.

From Figure 8 , it is observed that an $\mathrm{RDF}=50 \%$ is the optimal value for the factory. This RDF value yields not only the most balanced WIP in the matching area, but also the least amount of WIP in this critical area. This result provided the company with an initial perspective regarding the WIP condition of the matching area and demonstrated the importance of factory operation strategy for part fabrication.

WIP AT THE PART MATCHING AREA

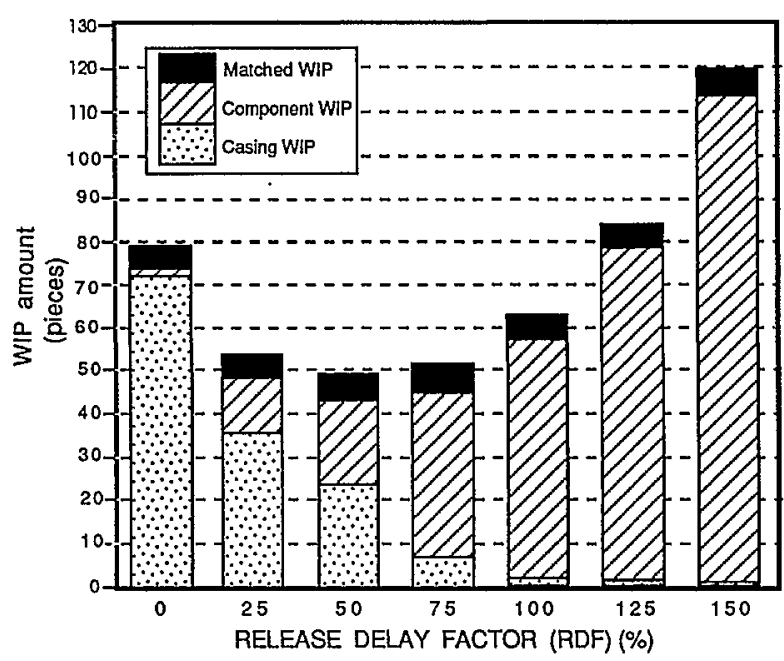

Figure 8: Effects of Job Release Delay Factor on Work-In-Process at the Part Matching Area 


\section{GRAPHICAL ANIMATION OF THE FACTORY OF THE FUTURE}

As the final step in the sequence of analysis and modeling studies, a graphical animation of the factory (similar to Figure 1) was created using the SIMAN/CINEMA linkage. Although, from a purely analytical point of view, the graphical animation does not provide more information than that of simulation, it is a powerful medium to verify and present the results of the analysis. Conditions of the bottleneck stations, the WIP status in the matching area, and the effects of the job release dates were all visible on the computer screen. This animation provided visual insights, endorsed the legitimacy of the numerical analysis, and gave the company's engineers a powerful communication medium to present the results to their management.

\section{BENEFITS AND EXPERIENCE GAINED FROM USING AN INTEGRATED SET OF MODELING SOFTWARE}

The team felt that four important benefits and experience arose from the analysis approach presented.

\subsection{REDUCTION IN MODELING TIME}

A direct benefit of using the integrated set of software was its short modeling time compared with other approaches. As Table 1 shows, about 80 man-hours were spent to complete the entire modeling. This significantly shortened modeling time allowed a successful completion of the analysis within the given time span. The team felt that the software linkages were the key in attaining such a short modeling time. For example, MANUPLAN's Lotus 1-2-3 interface simplified the building of an initial factory model, SimStarter allowed quick conversion of a MANUPLAN model into a SIMAN simulation model, and the SIMAN/CINEMA linkage made generation of animation easy. These software linkages eliminated unnecessary duplicate activities for modeling work, thereby minimizing modeling time required.

\subsection{USING THE RIGHT MODELING SOFTWARE AT THE RIGHT TIME}

Ability to use the right software at the right time is another benefit the team experienced for the integrated set of software. The team used Lotus 1-2-3 for data base manipulation, MANUPLAN for rough-cut capacity analysis, SIMAN for factory operation strategy, and CINEMA for visual presentation. The four software packages complemented each other quite well as the modeling and analysis progressed. Simulation could be used for rough-cut capacity planning but it is not appropriate for a large number of quick what-if questions which MANUPLAN does easily. (See computation time in Table 1) On the other hand SIMAN works well for the analysis of the real time factory operation strategy whereas MANUPLAN is not suited to this aspect. Lotus 1-2-3 has proven data base manipulation capability but does not perform dynamical analysis. Each software package has its own benefits and drawbacks. Thus it is essential to use the right software for a specific modeling and analysis purpose at the right time. The integrated set of modeling software used here fulfills this requirement by covering the entire activities in design and analysis of manufacturing systems .

\subsection{USE IN STUDYING DESIGN AND OPERATION OF MANUFACTURING SYSTEMS}

The third benefit is that both design and operational aspects of manufacturing systems were studied by using this integrated set of software. Manufacturing system performance can be maximized by superior system design coupled with efficient system operation. Therefore it is important to analyze these two aspects prior to implementing a planned manufacturing system on a shop floor. In this sense, the integrated set of software is very useful. MANUPLAN is used for rough-cut system design and SIMAN for detailed design. MANUPLAN can analyze rough-cut operational strategies such as lot-sizing while SIMAN can take care of detailed operational strategies such as part scheduling. Ability to handle both of these concerns from tough-cut level to detailed level is a great benefit provided by the integrated set of software.

MODELING AND ANALYSIS TIME

(Time unit : hours)

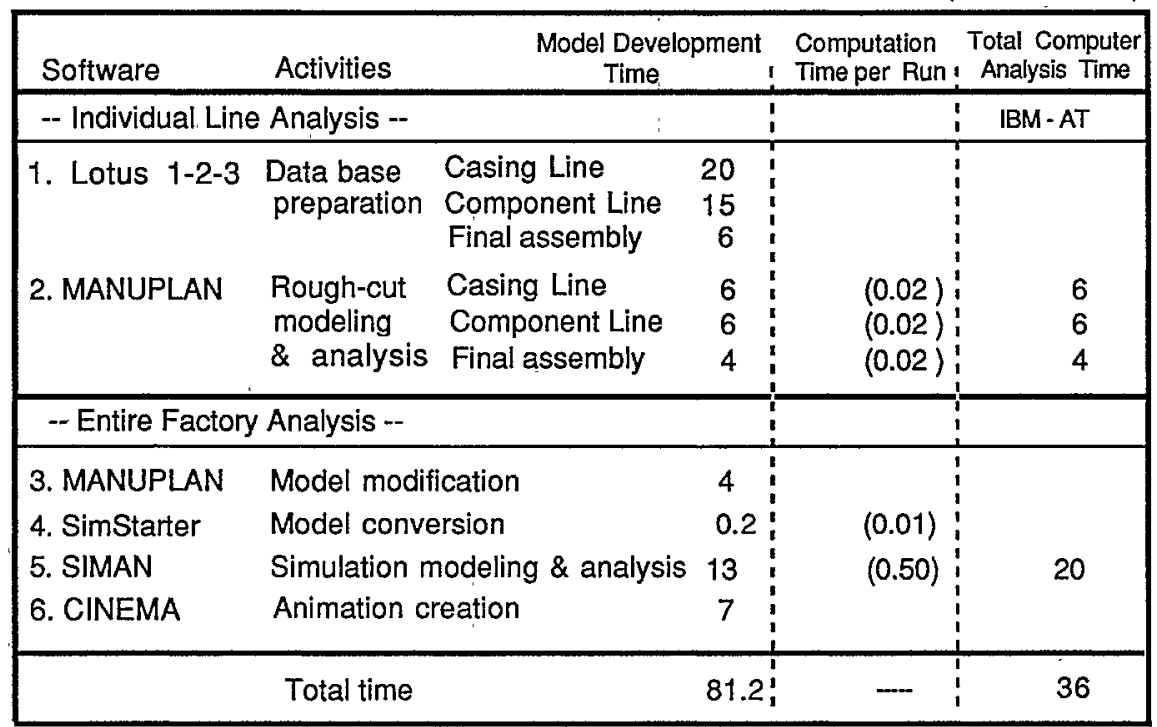

Note: MANUPLAN modeling times are longer than normal, since some members used MANUPLAN for the first time.

Table 1: Time Spent for Respective Modeling and Analysis Stages 


\subsection{SIMPLIFICATION OF SUBSEQUENT ANALYSIS STAGES}

Throughout the analysis the team experienced how each of the preceding analysis stages simplified the following work to be done. As Table 1 showed, almost $50 \%$ of modeling time was spent in data base preparation. This time was spent mainly in aggregation of the data base. As a result of this well thought-out aggregation strategy, the remaining modeling work could be completed in a short period of time. Although the data base was aggregated, the key issues of the new factory were fully analyzed and appropriate conclusions were drawn. Similarly, by doing initial capacity balancing using MANUPLAN, a lot of simulation run time was saved, creating more time to study operating strategies. A similar benefit was seen in another factory-of-the-future analysis done at IBM. [Brown (1988)]

\section{CONCLUSIONS}

Application of an integrated set of modeling software for the analysis of a 'factory of the future' was presented, with specia emphasis on the application procedure. Results of the analysis and benefits of using such modeling tools were also presented. Since factory configurations such as the one presented here are commonly found in today's manufacturing environment, the authors expect that the approach and experience presented in this paper should be helpful for persons working in design and analysis of manufacturing systems in other facilities.

\section{ACKNOWLEDGEMENTS}

The authors would like to express their sincere appreciation to Professors Rajan Suri and Jerry Sanders of the Industrial Engineering Department of the University of Wisconsin-Madison for their thoughtful suggestions and frequent encouragement to complete this project work. The authors appreciation is also extended to other members of the UW analysis team, Mr. Patrick Austin, Mr. Sudhakar Dharnipragada, and Mr. Michael Tomsicek. Their contributions to the project form a vital part of this paper.

This work was supported in part by National Science Foundation Grant No. ISI 8521292, General Motors Corporation under Research Agreement No. 30-8, and a grant from RTE Corporation.

\section{REFERENCES}

Anderson, K.R., Diehl, G.W., Shimizu, M., and Suri, R., (1988) Integrating spreadsheets, system modeling, and animation for rapid computer-aided design of manufacturing systems: Proceedings of the Sixth National Conference on University Programs in Computer-Aided Engineering, Design and Manufacturing (R.E.Fulton and J.I.Craig, eds), Georgia Institute of Technology, Atlanta, Georgia, 22-27

Brown, E. (1988) IBM combines rapid modeling technique and simulation to design PCB factory-of-the-future: Industrial Engineering, Vol.20-No.6, 23-26

Miles, T. and Sandowski, R. (1988) Animation design with CINEMA: Proceedings of the 1988 Winter Simulation Conference (forthcoming)

Pegden, C.D. (1987) Introduction to SIMAN: Systems Modeling Corporation

Shimizu, M. (1988) An integrated approach for rapid design and analysis of manufacturing systems: Technical Report, College of Engineering, University of Wisconsin - Madison, Madison, Wisconsin
Suri, R. (1988) RMT puts manufacturing at helm: Manufacturing Engineering, Vol.100-No.2, 41-44

Suri, R., Diehl, G.W., and Dean, R.R. (1986) Quick and easy manufacturing system analysis using MANUPLAN: Proceedings of the 1986 Annual International Industrial Engineering Conference, Institute of Industrial Engineers, Dallas, Texas, 195205

Suri, R. and Tomsicek, M. (1988) Rapid tools for manufacturing simulation and analysis: Proceedings of the 1988 Winter Simulation Conference (forthcoming)

\section{AUTHORS' BIOGRAPHY}

MASAMI SHIMIZU is a graduate student of the Manufacturing Systems Engineering Program at the University of Wisconsin-Madison. He is currently on leave from Mitsubishi Heavy Industries, Ltd of Japan where he is employed as a senior manufacturing engineer. His professional specialization includes manufacturing system design, design review for manufacturability, metal cutting, process planning, machine tool inspection, and quality control. His current interests are Computer Integrated Manufacturing, design and analysis using manufacturing systems modeling, and precision machining. He is a member of IIE, SME, APICS, the Japan Society of Precision Engineering, and the Japan Industrial Management Association.

DAVID VAN ZOEST is a graduate student of the Manufacturing Systems Engineering Program at the University of Wisconsin-Madison. He is currently teaching senior level laboratory courses in the manufacturing processes. His present interests includes modeling and design of manufacturing systems, advanced manufacturing processes, and application of modern manufacturing technologies to developing countries. He is a member of SME.

Masami Shimizu and David Van Zoest

Manufacturing Systems Engineering

University of Wisconsin - Madison

164 Mechanical Engineering Building

1513 University Avenue

Madison, Wisconsin 53706

(608)-262-4404 (Shimizu)

(608)-263-2423 (Van Zoest) 